\title{
Reserve Costs Allocation Model for Energy and Reserve Market Simulation
}

\author{
Tiago Pinto ${ }^{1,2}$, Amin Shokri Gazafroudi ${ }^{1}$, Francisco Prieto-Castrillo ${ }^{1}$, Gabriel Santos ${ }^{2}$, Francisco Silva ${ }^{2}$, \\ Juan Manuel Corchado ${ }^{1}$, Zita Vale ${ }^{2}$ \\ ${ }^{1}$ BISITE research group - University of Salamanca \\ Calle Espejo, s/n, 37007, Salamanca, España \\ \{tpinto, shokri, franciscop, corchado\}@usal.es \\ ${ }^{2}$ GECAD - Research Group on Intelligent Engineering and Computing for Advanced Innovation and Development, \\ Institute of Engineering - Polytechnic of Porto (ISEP/IPP) \\ Rua Dr. António Bernardino de Almeida, 431, 4200-072 Porto, Portugal \\ \{gajls, fspsa, zav\}@isep.ipp.pt
}

\begin{abstract}
This paper proposes a new model to allocate reserve costs among the involved players, considering the characteristics of the several entities, and the particular circumstances at each moment. The proposed model is integrated in the Multi-Agent Simulator of Competitive Electricity Markets (MASCEM), which enables complementing the multi-agent simulation of diverse electricity market models, by including the co-simulation of energy and reserve markets. In this context, the proposed model allows allocating the payment of reserve costs that result from the reserve market. A simulation based on real data from the Iberian electricity market - MIBEL, is presented. Simulation results show the advantages of the proposed model in sharing the reserve costs fairly and accordingly to the different circumstances. This work thus contributes the study of $\mathrm{nc} \ldots$ market models towards the evolution of power an en rosystems, with the objective of adapting current and future models to the new paradigm of high renewat energy generation penetration.
\end{abstract}

Index Terms-Electricity markets, MIBEL, Mv ti-Agent simulation, Reserve costs allocation.

\section{INTROD $\mathrm{CT}_{1} \mathrm{~N}$}

With the massive introc' iction of renewable based generation in power vistems, e ectricity markets are undergoing profound tra sfor tions [1]. These changes aim to cope with the partic 'ar characteristics of renewable generation (namely the var tion due to their dependency on natural resources) and to promote the increase of an active participation from the consumers' side. Such changes, however, turn the electricity market sector into a highly demanding and complex environment [2]. In order to cope with this evolving, complex and dynamic reality, electricity market simulators are being increasingly used as a promising solution to enable the involved players gaining experience to act in the frame of a changing economic, financial, and regulatory environment. There are several experiences that sustain that a multiagent system with adequate simulation

This work has received funding from the European Union's Horizon 2020 research and innovation program under the Marie Sklodowska-Curie grant agreement No 641794 (project DREAM-GO) and from FEDER Funds through COMPETE program and from National Funds through FCT under the project UID/EEA/00760/2013. abilities is suitable to simulat electricity markets [3-6], considering the complex inter ctiuns between the involved players. Some refer nce exa aples of electricity market simulators are AMГS (1, gent-based Modeling of Electricity Systems) [3], EN CA Elctricity Market Complex Adaptive System), [4] and M/ SCEM (Multi-Agent Simulator of Competitive E ctricity Markets) [5, 6]. These are important contribut ons out, in general, lack flexibility as they adopt a limi d amb r of market models and of players' meth toloysus. One of the most relevant limitations refers to n. stu $y$ and analysis of reserve markets, and their connection with c...rgy markets, especially considering the current and future scenario of high renewable generation penetration. In this scope, a relevant unsettled question is determining who should pay for the reserve costs.

In the literature, different works that propose models to allocate reserve costs have been presented. In [7], a method has been proposed to obtain optimal bidding of operating reserves in the sequential market clearing of the Spanish electricity market. The flexible Expected Energy Not Supplied (EENS) criteria and the load point reliability of customers have been presented to allocate the reserves, respectively in [8] and [9]. In [10], authors present the reserve cost allocation method through market agents based on the desired reliability level of electrical consumers. In [11], the reserve costs have been allocated between Distribution Companies (DisCos) based on their Value Of Lost Load (VOLL). A mechanism has been presented to determine the operating reserve and apportion the reserve costs between electrical customers and Generator Companies (GenCos) in [12]. In [13], a novel mechanism based on a decentralized approach has been introduced to allocate reserve costs between consumers, generating units and system operator in the simultaneous energy and reserve market clearing problem.

Moreover, different countries use different methods to allocate reserve costs. For example, in the UK electricity 
market, both GenCos and the electrical consumers are charged for reserve costs [14]. However, GenCos are in charge of paying the reserve costs in some electricity markets (e.g. Austria, Netherlands and Singapore) [15-17]. On the other hand, allocating the reserve costs between GenCos can affect negatively on the electrical consumers because GenCos increase their corresponding energy price to compensate their loss of reserve cost. Therefore, in Switzerland and most of the electricity markets in the world, the demand-side participants consumers or DisCos - oversee paying the reserve costs [18].

In this paper, a new method is proposed to allocate reserve costs between electrical customers, to support reserve market integration with day-ahead spot, intra-day and balancing markets. The proposed model is integrated in the MASCEM electricity market simulator, and its impact on the reserve market is studied. For this, a simulation scenario comprising real data from the Iberian electricity market - MIBEL [19] is generated. The simulation considers the day-ahead spot market, the intra-day market, and finally a reserve market, from which results the reserve price based on the total amount on EENS. The proposed reserve costs allocation model is then applied to determine who should pay for the reserve costs.

After this introductory section, section II presents an overview of MASCEM and section III describes the electricity market models that are used in this study. Section IV presents the proposed reserve costs allocation model formulation, and a case study is presented in section $\mathrm{V}$ to validate and assess the achieved results. Finally, section VI discusses the most relevant conclusions from this work.

\section{MASCEM SIMULATOR}

The MASCEM simulator has recently been res ucture [6], among many reasons, in order to guara tee the compliance with the FIPA (Foundation for Intel 1 go. + r sical Agents) standards [20], allowing the integratic with external platforms. FIPA is devoted to develop al pam open specifications that support interoperabil amo agents and agent-based applications [20]. Mv '-ag t systems using FIPA's standards should be able in "onerate, however, it does not mean that the agents an able to share useful information due to the er-nloyme. of lifferent ontologies.

Coping with FIPA $s^{+}$nu. ds meant implementing MASCEM's agent society JADE (Java Agent DEvelopment Framework) [21], a develo ment framework that simplifies the implementation of multi-agent systems, and supports the majority and most important FIPA specifications. This way MASCEM is able to interact with other multi-agent systems using a common language. However, so that messages and all the concerned concepts can be understood by different systems it is also necessary that they share a common vocabulary and semantics. To this end, ontologies are used, enabling the standardization of communications and interpretation of concepts between independent systems.

MASCEM includes only five different types of agents, besides the ones provided by JADE to control the user interfaces, and to manage communications, much like the previous version facilitators. The five agent types are:
- Main Agent - enables the user's interaction with the system. It is responsible for starting the market entities from the input file or user's interface; for converting the input data into the respective RDF knowledge bases and for sending them to the respective players and operators; for distributing the various agents by the machines available for the simulation, considering the machines' features and the agents' processing needs; and for properly killing the agents when the user decides to terminate the application;

- MIB Agent - it is responsible for reading the management information base of each machine, creating a report and sending it to the Main Agent so that it can decide which agents will move to each machine;

- Market Operator - regulates pool negotiations by validating and analysing the players' bids depending on the type of negotiation, and deter an the market price, the accepted and refused bids, an the onomical dispatch that will be sent to the system $0^{n}-$ to

- System Operatc (ISC) - examines the technical feasibility from the po er vorem point of view and solves congestion proble ns that ay arise. It is responsible for the system's secur y we as to assure that all conditions are met within the vsterin,

Pla er-represents buyer, seller or aggregator agents. On ( ne a d, may be a consumer or distribution company which participates in the EM in order to buy certain amounts Or ov er. On the other hand, it may simulate electricity producers or other entities able to sell energy in the market, or even aggregations of several entities.

These core agents allow a simple, yet effective electricity market simulation. More complex and advanced simulation studies are achieved through the collaboration between the different multi-agent systems, as presented in Figure 1.

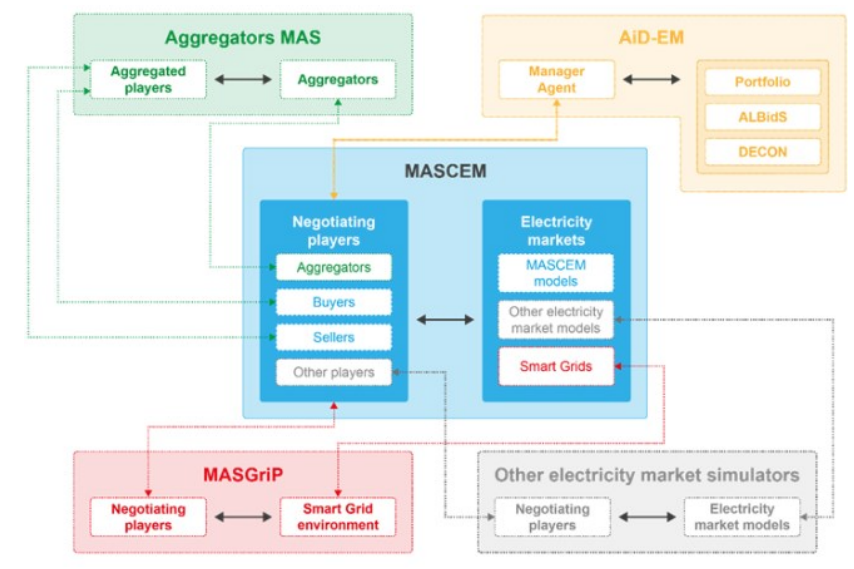

Figure 1. Collaboration between MASCEM and other multi-agent systems, from [6]

Regarding the market models, MASCEM allows the simulation of several market types: day-ahead pool (asymmetric or symmetric, with or without complex conditions), bilateral contracts, balancing market, forward markets and ancillary services. Hybrid simulations are also possible as a combination of the different market models. Other market types can be provided by different systems. 
Simulation scenarios in MASCEM are automatically defined, using the Realistic Scenario Generator (RealScen) [22]. RealScen uses real data that is available online, usually in market operators' websites. The gathered data concerns market proposals, including quantities and prices; accepted proposals and established market prices; proposals details; execution of physical bilateral contracts; statement outages, accumulated by unit type and technology; among others. By combining real extracted data with the data resulting from simulations, RealScen offers the possibility of generating scenarios for different types of electricity markets. Taking advantage on MASCEM's ability to simulate a broad range of different market mechanisms, this framework enables users to consider scenarios that are the representation of real markets of a specific region; or even consider different configurations, to test the operation of the same players under changed, thoroughly defined scenarios [22]. When summarized, yet still realistic scenarios are desired (in order to decrease simulations' execution time or facilitate the interpretation of results), data mining techniques are applied to define the players that act in each market. Real players are grouped according to their characteristics' similarity, resulting in a diversity of agent types that represent real market participants.

MASCEM is also integrated with AiD-EM (Adaptive Decision Support for Electricity Markets Negotiations), a decision support system that supports players decisions in their market negotiations. The AiD-EM system has been developed with the purpose of providing decision support to electricity market negotiating players. This system is composed by several distinct and independent decision support systems, each directed to the resolution of diffe ent specific problems. ALBidS (Adaptive Learning Bidding System) [23-25] focuses on the support o markc players' decisions when participating in auct n-based markets. The participation in bilateral contract nes tia on is supported by yet another multi-agent-based d cisior support system - DECON (Decision Suppor fo Ner contracts Negotiations) [26]. The multiagent $a_{1}$ roac of AiD-EM facilitates the interactions between $\mathrm{d}_{1}$ rent components and also the communication with ext nal agents, such as market players that make use of the do ision support.

\section{REGULATOR ELI TICITY MARKET MODELS}

As a result of the co stant evolution of the electricity market environment, and e inclusion and change in the operation and players' participation in the market, it became imperative for professionals in the area to entirely understand the markets' principles and how to evaluate their investment under such a competitive environment. The shared interest of regulators and market players in foreseeing market behaviour required a clear understanding of market principles, and the impact of power systems physics on market dynamics and vice-versa $[2,27]$. Additionally, a suitable understanding of the diversity of market types and regulatory models that have been introduced is critical for the success of involved players.

The typical electricity market environment in Europe usually consists of a day-ahead pool (symmetric or asymmetric) where energy for the following day is negotiated. Typically, a floor for bilateral contracts is also considered
[28]. Moreover, intraday markets are required to provide the means to renegotiate the previously traded power in order to meet the required adjustments towards the feasibility of the daily program and of the last scheduling [29]. Given the different market opportunities, each market player must decide whether to, and how to, participate in each market type.

In addition to players (the buyers and sellers who negotiate in the market), these markets also include the market and system operators. The market operator is the entity responsible for operating the market. It manages the pool by using a market-clearing tool which establishes the market price for each trading period and the accepted and refused selling and buying bids. On the other hand, the system operator is the entity responsible for the management of the transmission grid and its technical constraints. After the establishment of a contract, the agreement is communicated to the system operator, which analyses its tec 'nic. ' feasibility in the power system perspective; regardless 4 it beng established through bilateral contracts or throv s $\mathrm{n}, \mathrm{v}, \mathrm{p}$.

\section{A. Symmetric pool}

In the symme ic n ol he market price definition is based on a double au diu mer nanism, being therefore characterized by a competit $\eta$ between buyer and seller agents. This means that, in th o s pool, both buyers and sellers submit bids.

i he i rke operator orders the supply and demand offers: the su ply bids are sorted from the lowest price to the highest; an the demand bids are arranged from the highest price to the lowest. Hereinafter, the submitted bids compose the supply and demand step curves, and the market price is determined at the point in which both curves intersect. The market price is the value per MWh that each accepted consumer must pay to the respective supplier. The supply bids offering prices lower than the established market price will be accepted, as well as the demand bids offering prices higher than the market price. Depending on the demand, the last seller to trade, i.e. the one who establishes the market price, may not be able to negotiate all of its available supply, trading only partially. This process is repeated for each trading period of the day. Figure 2 illustrates the dispatch procedure of the symmetric pool.

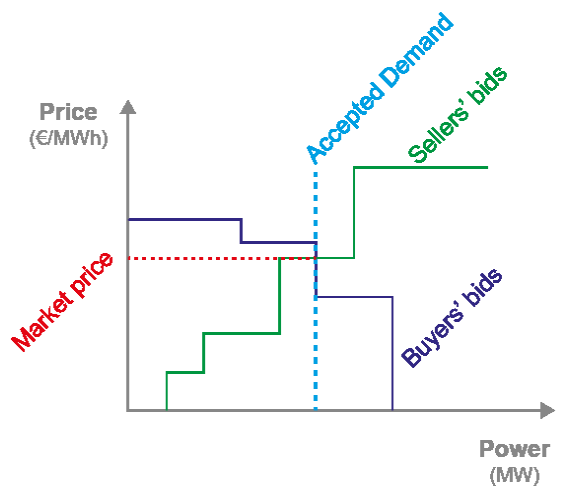

Figure 2. Symmetric pool, adapted from [6]

The efficiency of this market pool depends on the number of participating players, as well as on their bids provision. Players submitting their bids in this type of market reveal the existence of behaviours with price sensitive consumptions. 


\section{B. Asymmetric pool}

The asymmetric pool is exclusive to the submission of supply bids. In this type of pool, buyers do not submit their bids, only indicate an estimate of consumption. In this model the demand is considered inelastic, since it is assumed that buyers participating in it are willing to pay any price resulting from the market operation. In this market type, seller agents submit their bids and the market operator orders them from the lowest price to the highest. As for buyers, they only express their demand needs. Afterwards, the market operator accepts only the supply necessary to fulfil the demand. The price to be paid to all the accepted suppliers is determined by the last accepted bid, i.e. the market price. Figure 3 shows the dispatch procedure of the asymmetric pool, for each negotiation period. Market prices in this pool are highly influenced by the prices of sale bids and by the amount of demand.

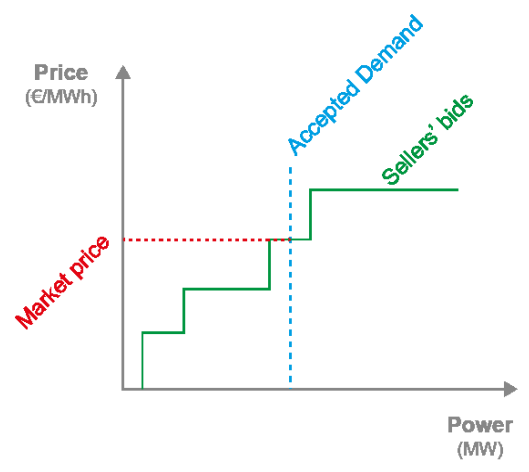

Figure 3. Asymmetric pool, adapted from [6]

\section{PROPOSED RESERVE COSTS ALLOCATION ML TEL}

Electrical customers play an essential role or needeu reserve in the power system. Providing electrical de. and of consumers and reliability of the power system are vo main reasons why reserve is needed. Hence, Demanc Facts is (DFs) of electrical consumers that are define $f_{c}$ th firs rime in [9] are considered in this paper, i. or ar to express the corresponding reliability level of 'Isto ers. Effects of electrical demand and Expected Enc ry $\wedge$ Supplied (EENS), as a reliability criteria, are const ered $1 \mathrm{DF}$ as in (1):

$$
D F_{j t}=\frac{L}{L_{j t}}
$$

where $L_{j t}$ represents the total load of consumer $j$ in time $t$. Customers are classified into different groups based on their DFs as seen in (2). A total number of $M$ groups is considered. In this case, $D F_{j t}^{1}$ has the highest corresponding $\mathrm{DF}$ and lowest corresponding reliability level. Hence, $\Delta D F_{j t}$ is defined as in (3), as a difference between $D F_{j t}^{1}$ and $D F_{j t}$.

$$
\begin{gathered}
D F_{j t}^{M} \leq \cdots \leq D F_{j t}^{2} \leq D F_{j t}^{1} \\
\Delta D F_{j t}=D F_{j t}^{1}-D F_{j t}
\end{gathered}
$$

As highlighted, when more reserve is needed to maintain system's reliability level, the reserve costs will be increased. This increment of reserve costs should be paid by customers who have their corresponding reliability level $\triangle D F$ higher than others. Therefore, the portion of electrical customers to pay the cost of reserve $(R C)$ is achieved by (4).

$$
R C_{j \mathrm{t}}=\frac{\Delta D F_{j t}}{\sum_{i=1}^{N_{2}} \Delta D F_{j t}} \times R C_{\mathrm{t}}
$$

As a result from this model, each customer who has higher $\triangle D F$ is allocated to pay more reserve costs. Moreover, electrical consumers who have highest DF are not in charge of paying reserve costs based on this proposed method.

\section{CASE STUdy}

This case study aims at assessing the envisaged energy and reserve market simulation, using the proposed reserve cost allocation model, through its integration in the MASCEM simulator. The case study consi ers se simulation of the dayahead electricity market of ML. EL, which is complemented by the intra-day market. in a llowing step, the reserve market is executed, con iderin; an asymmetric auction, in which generators bid $t_{1}$. ir n...ulable capacity for reserve and corresponding pr e. Con dering the total expected energy non-supplied, 1 - wery cost is achieved from this market. The payment $f$ this ust is then determined by the proposed reserve cr or 'loc tion model.

I or to set up this market environment, a simulation scena o was generated using RealScen. The considered su ar concerns 48 market negotiating agents. The main decision factor for the simulated agents' representation is the real players' competitiveness, i.e. their bid prices' approximation to the established electricity market price. The objective is to represent the electricity market environment, which includes hundreds of players negotiating in each negotiating period, in a reduced simulated scenario that reflects the reality in the fullest possible extent. In order to enable the simulator to represent the reality in a summarized way, the automatic data extraction tool has been used to gather data from the Iberian electricity market - MIBEL [19]. The simulated scenarios refer to the 24 hourly periods of the dayahead spot market, in one negotiating day - June, $1^{\text {st }} 2012$. The simulated agents consider the log of real players' bids to the market during this day, in order to create their own action portfolio in the market. Figure 4 shows the electricity market price and total amount of transacted and non-transacted energy in the day-ahead spot market simulation, during the 24 hourly periods of the considered simulation day.

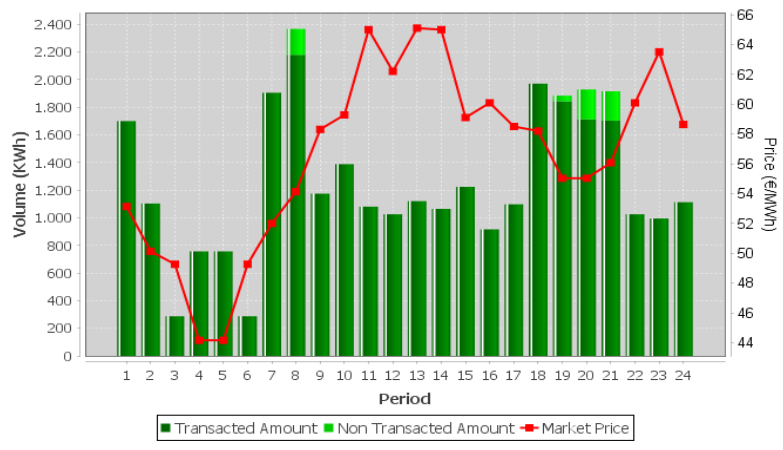

Figure 4. Day-ahead market results 
From Figure 4 it is visible that the day-ahead market prices in the considered day range from $44 € / \mathrm{MWh}$ to $66 € / \mathrm{MWh}$. It is also visible that most of the available energy has been transacted, with the exception of 4 periods. One of these is period 20, for which Figure 5 shows some details, namely referring to the demand and supply bid curves, which are composed by the price and volume offers made by the participating agents.

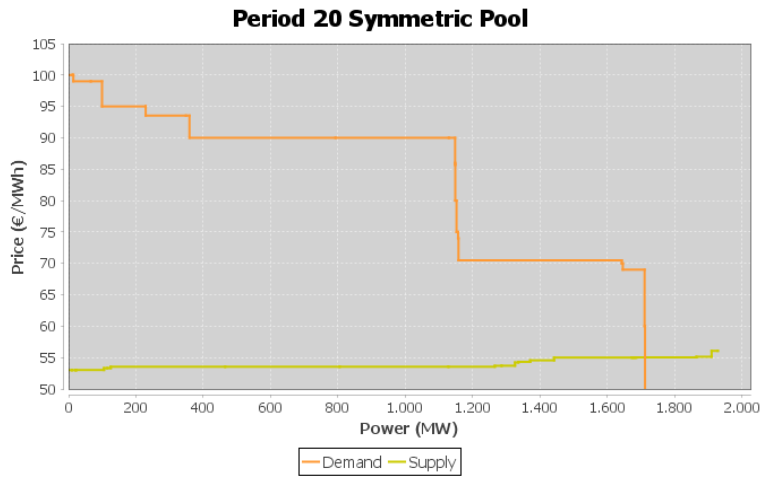

Figure 5. Day-ahead spot market bid curves for period 20

From Figure 5 one can see that the point where the demand and supply curves intersect in this period corresponds to the price of $55,03 € / \mathrm{MWh}$ - the established market price for this period. It is also visible that some supply is not dispatched, namely the amount that is associated to prices higher than the market price.

Departing from the day-ahead spot market results considering the variations in expected consump on an generation from the previous day towards the delivery time, the intra-day market is executed. This market, according to the MIBEL rules [19], considers 6 market sessions, which cover all the periods of the delivery day, and which are executed in different time horizons. Based on the results of the intra-day market and on the individual strategies and goals, generators submit their bids to the reserve market, which are composed by the volume of energy that is available for reserve, and the associated price. In this case, the market runs as an asymmetric auction, considering only the bids from generators. The reserve market price is established depending on the amount of reserve that is required. Figure 6 shows the supply bid curve for the asymmetric reserve market auction, still referring to hourly period 20.

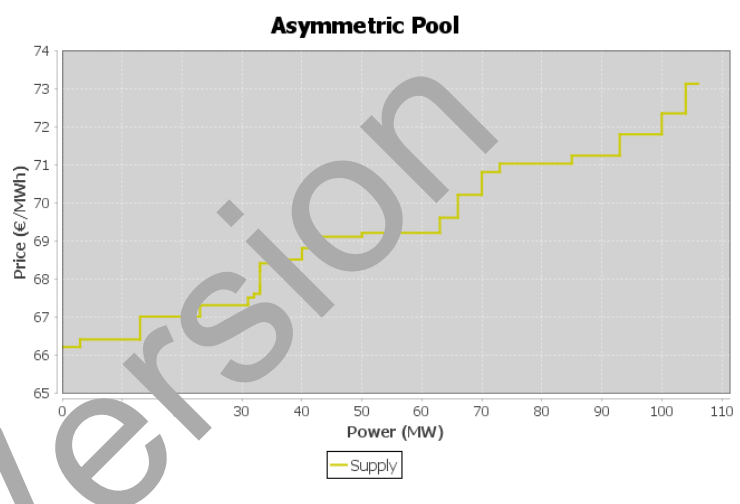

Figure 6. Reserve market supply bid curve for period 20

TABLE I. R sERVE C $\triangle$ S ALLOCATION RESULTS

\begin{tabular}{|c|c|c|c|c|c|c|}
\hline Consumer & LOAD (MWh) & ENS (MWh) & DF & $\Delta \mathrm{DF}$ & Group & $\mathbf{R C}(€)$ \\
\hline Buyer 1 & 1,3 & & 1 & 0 & 1 & 0 \\
\hline Buyer 2 & 64,7 & & 0,066461 & 0,933539 & 8 & 256,6547446 \\
\hline Buyer 3 & 3,2 & 3,2 & 1 & 0 & 1 & 0 \\
\hline Buyer 4 & 485 & 12,3 & 0,025361 & 0,974639 & 14 & 267,9541594 \\
\hline Buyer 5 & 1,2 & 0 & 0 & 1 & 15 & 274,9265227 \\
\hline Buyer 6 & & 0,4 & 0,129032 & 0,870968 & 3 & 239,4521327 \\
\hline Buyer 7 & & 0 & 0 & 1 & 15 & 274,9265227 \\
\hline Buyer 8 & $\jmath, 1$ & 0 & 0 & 1 & 15 & 274,9265227 \\
\hline Buyer 9 & 3,7 & 0,6 & 0,162162 & 0,837838 & 2 & 230,3438434 \\
\hline Buyer 10 & 0,4 & 0 & 0 & 1 & 15 & 274,9265227 \\
\hline Buyer 11 & 0,1 & 0 & 0 & 1 & 15 & 274,9265227 \\
\hline Buyer 12 & 433,7 & 21,3 & 0,049112 & 0,950888 & 10 & 261,4242517 \\
\hline Buyer 13 & 336,8 & 16,7 & 0,049584 & 0,950416 & 9 & 261,2944772 \\
\hline Buyer 14 & 18,6 & 2,3 & 0,123656 & 0,876344 & 4 & 240,9302323 \\
\hline Buyer 15 & 119,7 & 4,6 & 0,038429 & 0,961571 & 12 & 264,3612595 \\
\hline Buyer 16 & 10,4 & 1,2 & 0,115385 & 0,884615 & 5 & 243,2042316 \\
\hline Buyer 17 & 129,3 & 8,7 & 0,067285 & 0,932715 & 7 & 256,4279864 \\
\hline Buyer 18 & 0,4 & 0 & 0 & 1 & 15 & 274,9265227 \\
\hline Buyer 19 & 51,9 & 1,6 & 0,030829 & 0,969171 & 13 & 266,4509459 \\
\hline Buyer 20 & 33,3 & 1,4 & 0,042042 & 0,957958 & 11 & 263,3680503 \\
\hline Buyer 21 & 9,6 & 1,1 & 0,114583 & 0,885417 & 6 & 243,4245253 \\
\hline Buyer 22 & 3,1 & 0 & 0 & 1 & 15 & 274,9265227 \\
\hline Buyer 23 & 0,9 & 0 & 0 & 1 & 15 & 274,9265227 \\
\hline Buyer 24 & 0,5 & 0 & 0 & 1 & 15 & 274,9265227 \\
\hline
\end{tabular}


Figure 6 shows the bids that have been submitted to the reserve market by the several generators. The total energy nosupplied in this scenario has been of $81 \mathrm{MWh}$. From Figure 6 it is possible to verify that this volume represents a reserve market price of $71,23 € / \mathrm{MWh}$, which leads to a total reserve cost of $5769,63 €$ in this period. The proposed reserve costs allocation model is applied to determine who should pay for this reserve cost. Table I shows the results from the reserve costs allocation model, considering $M=15$.

As seen in Table I, reserve cost is allocated between consumers based on the proposed method. In this way, consumers 1 and 3 have their $D F$ equal to one, and are therefore not responsible for paying reserve costs. Also, their $D F_{j t}^{1}$ is equal to zero. Table I shows that customers should pay more reserve costs when they have higher $\Delta D F_{j t}$. Hence, in this case study, the allocated reserve costs related to consumers $5,7,8,10,11,18,22,23$ and 24 is highest because their corresponding $\Delta D F_{j t}$ has the highest amount in the system.

\section{CONCLUSIONS}

This paper addresses the simulation of energy and reserve markets, using a proposed model for reserve costs allocation as means to distribute the reserve costs that result from the market negotiations. Results from a case study based on real data from MIBEL, simulated using MASCEM, show that the proposed reserve costs allocation model enables distributing the reserve costs among the most appropriate consumers, in order to guarantee the payment for the reserve that is negotiated in the reserve market. The presented simulat ons also enable considering the proposed approach as a pi mi solution for the evolution of electricity markets.

Future work will consider alternative mode ${ }^{1}$ for eserve costs allocation, as well as the assessment of other possible solutions for reserve market mechanisms, in or 'er t smooth the connection with the current day read, intra-day and balancing market models.

[1] F. Sioshansi Evolution o whal Elc y Markets - New paradigms, new challenges, new appr ache ademic Press, 2013

[2] D. Biggar and M. Hesam (Eds.) The Economics of Electricity Markets. Wiley, 1st edition, eptember 22, 2014

[3] H. Li, and L. Tesfatsion, "D velopment of Open Source Software for Power Market Research: The AMES Test Bed", Journal of Energy Markets, vol. 2, no. 2, pp. 111-128, 2009

[4] V. Koritarov., "Real-World Market Representation with Agents: Modeling the Electricity Market as a Complex Adaptive System with an Agent-Based Approach", IEEE Power \& Energy magazine, pp. 3946, 2004

[5] I. Praça, C. Ramos, Z. Vale, and M. Cordeiro, "MASCEM: A MultiAgent System that Simulates Competitive Electricity Markets", IEEE Intelligent Systems, vol. 18, No. 6, pp. 54-60, Special Issue on Agents and Markets, 2003.

[6] G. Santos, T. Pinto, I. Praça, Z. Vale, "MASCEM: Optimizing the performance of a multi-agent system", Energy, vol. 111, pp. 513-524, September 2016

[7] F. A. Campos, A. M. S. Roque, E. F. Sánchez-Úbeda, and J. P. González, "Strategic Bidding in Secondary Reserve Markets", IEEE Trans. Power Syst., vol. 31, vo. 4, pp. 2847-2856, July 2016.
[8] M. Najafi, M. Ehsan, M. Fotuhi-Firuzabad, A. Akhavein, K. Afshar. "Optimal reserve capacity allocation with consideration of customer reliability requirements", Energy, vol. 35, no. 9, pp. 3883- 3890, Sep. 2010.

[9] P. Wang, L. Goel, "Reliability- based reserve management in a bilateral power market", Elect. Power Syst. Res., vol. 67, no. 3, pp. 185- 189, Dec. 2003.

[10] A. Ahmadi-Khatir, M. Fotuhi-Firuzabad, L. Goel, "Customer choice of reliability in spinning reserve procurement and cost allocation using well-being analysis", Elect. Power Syst. Res., vol. 79, no. 10, pp. 14311440, Oct. 2009.

[11] A. Ahmadi-Khatir, R. Cherkaoui, "A probabilistic spinning reserve market model considering Disco' different value of lost load", Elect. Power Syst. Res., vol. 81, no. 4, pp. 862- 872, Apr. 2011.

[12] A. Shokri Gazafroudi, K. Afshar, N. Bigdeli, "Assessing the operating reserves and costs with considering customer choice and wind power uncertainty in pool-based power market", Inter. J. of Elec. Power \& Energy Syst., vol. 67, pp. 202-215, May 2015.

[13] T. W. Haring, D. S. Kirschen, G. Andersson, "Efficient allocation of balancing and ramping costs", P- Power Syst. Comput. Conf. (PSCC), 18-22, Aug. 2014.

[14] National Grid Operation Manuals. Available from: http://www.nationalgrid.ce a/ un (on. e).

[15] G. Strbac, D.S. Ki chen "Who hould pay for reserve?", Electr. J., vol. 13 , no. 8, pp. 32-3 $\left(+20^{\prime} \mathrm{J}\right.$.

[16] B. Kirby, E. Hirst "Allo ting the costs of contingency reserves", The Electr. J., vol. 1', no in $p_{\mathrm{r}}$. 39-47, Dec. 2003.

[17] L.M. Xia, H 3. Uoi, , Bai, "A probabilistic reserve with zero-sum settlement 5 eme", ${ }^{2}$ Trans. Power Syst., vol. 20, no. 2, pp. $993-$ $1000, M-20$

[18] Anci ary servicus Unbundling Electricity Products - An Emerging Tark + Rep t EURO Electric, 2004.

[19] I IBEL Iberian Electricity Market Operator, homepage, ht. ://www.omie.es, accessed on March 2017

[20] dation for Intelligent Physical Agents (FIPA). Agent management specification, 2002.

[21] Java Agent Development Framework (JADE): Available: http://jade.tilab.com

22] B. Teixeira, F. Silva, T. Pinto, I. Praça, G. Santos, and Z. Vale, "Data Mining Approach to support the Generation of Realistic Scenarios for Multi-Agent simulation of Electricity Markets," in Intelligent Agents (IA), 2014 IEEE Symposium on, 2014, pp. 8-15.

[23] T. Pinto, Z. Vale, F. Rodrigues, H.o Morais, I. Praça, "Bid Definition Method for Electricity Markets Based on an Adaptive Multiagent System", Advances on Practical Applications of Agents and Multiagent Systems, vol. 88, pp. 309-316, Springer Berlin Heidelberg, April 2011

[24] T. Pinto, Z. Vale, F. Rodrigues, H. Morais, I. Praça, "'Strategic Bidding Methodology for Electricity Markets using Adaptive Learning, Modern Approaches in Applied Intelligence, vol. 6704, pp. 490-500, K. Mehrotra, C.Mohan, J. Oh, P. Varshney, M. Ali (Eds.), Springer Berlin / Heidelberg, 2011"

[25] T. Pinto, Z. Vale, F. Rodrigues, I. Praça, H. Morais, "Multiagent System for Adaptive Strategy Formulation in Electricity Markets", IA 2011 - International Symposium on Intelligent Agents at IEEE SSCI 2011 (IEEE Symposium Series on Computational Intelligence), Paris, France, April 11-15, 2011

[26] T. Pinto, Z. Vale, I. Praça, E. J. Solteiro Pires, F. Lopes, "Decision Support for Energy Contracts Negotiation with Game Theory and Adaptive Learning", Energies, MDPI, vol. 8, no.9, 9817-9842, September 2015,

[27] L. Meeus, K. Purchala and R. Belmans. Development of the Internal Electricity Market in Europe. The Electricity Journal, vol. 18, no. 6, pp. 25-35, 2005.

[28] "Electricity Advisory Committee, Keeping the Lights On in a New World. January 2009. [Online]. Available: http://energy.gov/sites/prod/files/oeprod/DocumentsandMedia/adequac y_report_01-09-09.pdf, accessed on March 2017"

[29] G. Santos, T. Pinto, Z. Vale, H. Morais and I. Praça, "Balancing Market Integration in MASCEM Electricity Market Simulator". IEEE Power and Energy Society General Meeting 2012, San Diego CA, USA, July $22-26,2012$ 